\title{
A full multigrid solution of control-constrained Cauchy-Riemann optimal control problems
}

\author{
M. M. Butt* $\quad$ A. Borzi ${ }^{\dagger}$
}

\begin{abstract}
The formulation and full-multigrid solution of control-constrained CauchyRiemann optimal control problems is presented. A constrained distributed control mechanism through divergence and curl sources is considered with mixed boundary conditions. The corresponding optimal solutions are obtained solving a Cauchy-Riemann optimality system consisting of four first-order partial differential equations and two inequality constraints. For the solution of the optimality system, staggered grids and a full-multigrid scheme are considered. The proposed full-multigrid method is based on a coarsening strategy by a factor of three that results in a nested hierarchy of staggered grids. The smoothing procedure consists of a distributed-Gauss-Seidel scheme for the state and adjoint equations and a projected gradient step for the controls. Numerical results validate the effectiveness of the proposed approach.
\end{abstract}

Key words: Cauchy-Riemann equations, constrained optimal control problems, staggered grids, finite differences, full multigrid method

AMS Classification: 35Q93, 49K20, 65N55.

\section{Introduction}

The purpose of control of partial differential equations (PDE) models addresses the need to define ways of how optimally change and influence many application systems to meet a desired target. While optimization with second-order PDEs has received much attention $[11,15]$, much less is known on optimization problems with first-order PDE models. We focus on the Cauchy-Riemann (CR) equations as representative elliptic first-order PDE systems and prototype of all div-curl systems. In particular, CR systems govern the velocity field of subsonic compressible inviscid flows [14, 17].

Our aim is to discuss the formulation and full multigrid solution of controlconstrained CR control problems. We formulate constrained CR control problems based on the optimization framework provided by the infinite-dimensional PDE optimal control theory $[11,12,15]$. In this framework, we consider a governing CauchyRiemann system with Dirichlet and Neumann boundary conditions and choose a

\footnotetext{
*Institut für Mathematik und Wissenschaftliches Rechnen, Karl-Franzens-Universität Graz, Heinrichstr. 36, 8010 Graz, Austria (muhammad.butt@edu.uni-graz .at) and Department of Mathematics, GC University, Katchery Road, Lahore 54000, Pakistan.

${ }^{\dagger}$ Institut für Mathematik, Universität Würzburg, Am Hubland, 97074 Würzburg, Germany (alfio.borzi@uni-wuerzburg.de).
} 
control mechanism of distributed type through div- and curl-source terms and a tracking criterion defining the objective of the control and including the cost of its action. Our optimal control problem is then formulated as the minimization of the tracking objective under the constraint given by the CR equations and control constraints. This work extends previous results on unconstrained-control CR optimization problems on staggered grids [1] and on a Galerkin-least-squares method for solving first-order elliptic control problems [8].

In the next section, we discuss a set of Cauchy-Riemann equations in twodimensional spatial domains with an appropriate functional setting. In Section 3, we introduce control-constrained Cauchy-Riemann optimal control problems and prove existence and uniqueness of optimal solutions. In particular, we discuss the characterization of these solutions as solutions to optimality systems consisting of four first-order PDE equations and two inequality constraints. In Section 4, we present a second-order approximation using staggered grids on non-uniform meshes. In our approach a natural collocation of the optimization variables is obtained that avoids interpolation of variables in the setup of the discretized CR optimality system. In Section 5, we illustrate our full multigrid method for solving the optimality system. It is shown that a coarsening by a factor of three of the mesh sizes is advantageous to solve the optimality system on a hierarchy staggered grids. In our scheme, a nested hierarchy is obtained. This results in more accurate and easy to implement inter-grid transfer operators. Further, we develop distributed-Gauss-Seidel relaxation schemes where the control functions are updated using a projected gradient-based approach. In Section 6, we report results of numerical experiments to validate our multigrid solution to control-constrained CR optimal control problems. These results demonstrated the ability of the full multigrid scheme to solve the CR optimality system for different optimization parameters with mesh-independent convergence factors. A section of conclusion completes this work.

\section{Cauchy-Riemann problem}

We consider a two-dimensional computational domain $\Omega \subset \mathbb{R}^{2}$ with $(x, y)$-coordinates and piecewise smooth boundary. We denote with $\mathbf{n}=\left(n_{x}, n_{y}\right)$ the unit outward normal vector to the boundary. Let $H^{1}(\Omega)$ denotes the Sobolev space of order one of real valued functions defined on $\Omega$. The space $H^{1}(\Omega)$ is equipped with the Sobolev norm $\|\cdot\|_{1}$ and associated seminorm $|\cdot|_{1}$. Moreover, we need the $L^{2}(\Omega)$ space and corresponding norm induced by the $L^{2}(\Omega)$ inner product $(\cdot, \cdot)$. A similar notation is used to denote norms in the product spaces $\mathbf{H}^{1}(\Omega)=H^{1}(\Omega) \times H^{1}(\Omega)$. The inner product in $\mathbf{L}^{2}(\Omega)=L^{2}(\Omega) \times L^{2}(\Omega)$ is also denoted by $(\cdot, \cdot)$.

Let $f, g \in L^{2}(\Omega)$, and $P(f, g)$ denotes the following CR problem or div-curl system in two-dimensions

$$
\begin{aligned}
\operatorname{div} \mathbf{w} & =f \text { in } \Omega, \\
\operatorname{curl} \mathbf{w} & =g \text { in } \Omega,
\end{aligned}
$$

where $\mathbf{w}=(u, v)$ are the state variables, $\mathbf{b}=(f, g)$ are the divergence-source and curl-source terms, and

$$
\begin{aligned}
\operatorname{div} \mathbf{w} & :=\partial_{x} u+\partial_{y} v \\
\operatorname{curl} \mathbf{w} & :=\partial_{x} v-\partial_{y} u
\end{aligned}
$$


subject to the following mixed boundary conditions

$$
\begin{aligned}
\mathbf{w} \times \mathbf{n}=0 & \text { on } \Gamma_{D}, \\
\mathbf{w} \cdot \mathbf{n}=0 & \text { on } \Gamma_{N}=\partial \Omega \backslash \bar{\Gamma}_{D},
\end{aligned}
$$

that is, $u n_{y}-v n_{x}=0$ on $\Gamma_{D}$ and $u n_{x}+v n_{y}=0$ on $\Gamma_{N}$. Here, $\Gamma_{D}$ and $\Gamma_{N}$ denote Dirichlet and Neumann boundaries, respectively.

Interpreting $\mathbf{w}$ as a velocity vector field in $\Omega$, we can see that the Dirichlet boundary conditions correspond to the assignment of the tangential velocity on the boundary, while on the Neumann boundary the normal component of the velocity is assigned. It is not a restriction to consider homogeneous boundary conditions since a non homogeneous problem can be transformed to an homogeneous one; see [18].

The CR problem (1)-(4) is well-posed and the following holds [1]

$$
\|\mathbf{w}\|_{\mathbf{H}^{1}(\Omega)} \leq c\|\mathbf{b}\|_{\mathbf{L}^{2}(\Omega)},
$$

where $c$ is a real constant. Moreover, the map

$$
\mathbf{L}^{2}(\Omega) \ni \mathbf{b} \mapsto \mathbf{w}(\mathbf{b}) \in \mathbf{H}^{1}(\Omega),
$$

defined by the unique solution of the div-curl system is continuous and Fréchet differentiable [8].

In the following, it is also convenient to consider a general three-dimensional setting. In this case, we write (1) and (2) in a differential vector notation

$$
\begin{array}{r}
\nabla \cdot \mathbf{w}=f \quad \text { in } \quad \Omega, \\
\nabla \times \mathbf{w}+\nabla \phi=\mathbf{g} \quad \text { in } \quad \Omega,
\end{array}
$$

where $\mathbf{w}=(u, v, w)$ and $\phi=\phi(x, y, z)$ is a scalar function that must be added to have a well-defined set of four first-order partial differential equations; see [8]. Additional conditions on $\phi$ are required that depend on the boundary condition settings. In the next section, we show that $\phi$ is actually a zero function with the following boundary conditions of mixed type

$$
\begin{aligned}
\mathbf{w} \times \mathbf{n}=0 & \text { on } \Gamma_{D}, \\
\mathbf{w} \cdot \mathbf{n}=0 & \text { on } \Gamma_{N}=\partial \Omega \backslash \bar{\Gamma}_{D},
\end{aligned}
$$

where $\mathbf{n}=\left(n_{x}, n_{y}, n_{z}\right)$. Notice that the three-dimensional setting reduces to the twodimensional case assuming that all variables do not depend on the third $z$-coordinate in $\Omega \subset \mathbb{R}^{3}$.

\section{Control-constrained CR optimal control problems}

We consider the following constrained CR optimal control problem

$$
\min J(u, v, f, g):=\frac{1}{2}\|\mathbf{w}-\mathbf{W}\|_{\mathbf{L}^{2}(\Omega)}^{2}+\frac{\alpha}{2}\|f\|_{L^{2}(\Omega)}^{2}+\frac{\beta}{2}\|g\|_{L^{2}(\Omega)}^{2},
$$

subject to $\mathbf{b}=(f, g) \in \mathbf{B}_{a d}$ and

$$
\begin{aligned}
\operatorname{div} \mathbf{w} & =f \\
\operatorname{curl} \mathbf{w} & =g,
\end{aligned}
$$


with mixed boundary conditions

$$
\begin{aligned}
\mathbf{w} \times \mathbf{n}=0 & \text { on } \Gamma_{D} \\
\mathbf{w} \cdot \mathbf{n}=0 & \text { on } \quad \Gamma_{N}=\partial \Omega \backslash \bar{\Gamma}_{D},
\end{aligned}
$$

We denote with

$$
\mathbf{B}_{a d}=\left\{(f, g) \in \mathbf{L}^{2}(\Omega): \underline{f} \leq f \leq \bar{f} \text { and } \underline{g} \leq g \leq \bar{g} \text { a.e. in } \Omega\right\},
$$

the nonempty, closed, and convex subset of $\mathbf{L}^{2}(\Omega)$ of admissible controls. We choose $\{\underline{f}, \bar{f}, \underline{g}, \bar{g}\} \subset L^{\infty}(\Omega)$. We denote with $\mathbf{W}=(U, V) \in \mathbf{L}^{2}(\Omega)$ the target functions, and $\alpha, \beta>0$ are the weights of the costs of the controls of $f$ and $g$ respectively.

Notice that the objective function $J$ is convex and the constraints are linear. Therefore existence of a unique solution to the constrained CR optimal control problem (9)-(13) and its characterization can be shown based on classical techniques $[11,12,15]$.

For convenience, we consider a three-dimensional setting and define the Lagrangian function as follows

$$
\mathcal{L}=\mathcal{L}(u, v, p, q, \phi, f, g)=J(u, v, f, g)+(\nabla \cdot \mathbf{w}-f, p)+(\nabla \times \mathbf{w}+\nabla \phi-\mathbf{g}, \mathbf{q}) .
$$

In this formulation, the unique solution to the optimal control problem satisfies the extrema conditions for the Lagrangian function, that are the first-order optimality conditions. Therefore, we have to evaluate the Fréchet derivatives of $\mathcal{L}$ with respect to the optimization variables. We have

$$
\begin{aligned}
\left(\nabla_{p} \mathcal{L}, \delta p\right) & =(\nabla \cdot \mathbf{w}-f, \delta p), \\
\left(\nabla_{q} \mathcal{L}, \delta \mathbf{q}\right) & =(\nabla \times \mathbf{w}+\nabla \phi-\mathbf{g}, \delta \mathbf{q}), \\
\left(\nabla_{f} \mathcal{L}, \tilde{f}-f\right) & =(\alpha f-p, \tilde{f}-f), \\
\left(\nabla_{g} \mathcal{L}, \tilde{\mathbf{g}}-\mathbf{g}\right) & =(\beta \mathbf{g}-\mathbf{q}, \tilde{\mathbf{g}}-\mathbf{g}) .
\end{aligned}
$$

We also have that

$$
\left(\nabla_{\phi} \mathcal{L}, \delta \phi\right)=(\nabla \delta \phi, \mathbf{q})=-\int_{\Omega}(\nabla \cdot \mathbf{q}) \delta \phi d x+\int_{\partial \Omega} \delta \phi(\mathbf{q} \cdot \mathbf{n}) d s .
$$

and

$$
\begin{aligned}
\left(\nabla_{\mathbf{w}} \mathcal{L}, \delta \mathbf{w}\right) & =(\mathbf{w}-\mathbf{W}, \delta \mathbf{w})+(\nabla \cdot \delta \mathbf{w}, p)+(\nabla \times \delta \mathbf{w}, \mathbf{q}) \\
& =(\mathbf{w}-\mathbf{W}, \delta \mathbf{w})-(\nabla p, \delta \mathbf{w})+\int_{\partial \Omega} p(\delta \mathbf{w} \cdot \mathbf{n}) d s \\
& -(\nabla \times \mathbf{q}, \delta \mathbf{w})+\int_{\partial \Omega}(\mathbf{q} \times \mathbf{n}) \cdot \delta \mathbf{w} d s
\end{aligned}
$$

Next, we obtain the following equations

$$
\begin{aligned}
\nabla \cdot \mathbf{w}-f & =0 \\
\nabla \times \mathbf{w}+\nabla \phi-\mathbf{g} & =0 \\
(\alpha f-p, \tilde{f}-f) & \geq 0 \\
(\beta \mathbf{g}-\mathbf{q}, \tilde{\mathbf{g}}-\mathbf{g}) & \geq 0 \text { for all }(\tilde{f}, \tilde{\mathbf{g}}) \in \mathbf{B}_{a d} .
\end{aligned}
$$


Requiring $\left(\nabla_{\phi} \mathcal{L}, \delta \phi\right)=0$ for any $\delta \phi \in H^{1}(\Omega)$, we have

$$
\nabla \cdot \mathbf{q}=0 \quad \text { in } \Omega .
$$

Further, we require $\left(\nabla_{\mathbf{w}} \mathcal{L}, \delta \mathbf{w}\right)=0$ for all $\delta \mathbf{w} \in \mathbf{H}^{1}(\Omega)$ that satisfy the homogeneous boundary conditions (12)-(13). Since $\delta \mathbf{w} \cdot \mathbf{n}=0$ on $\Gamma_{N}$, we must have $p=0$ on $\Gamma_{D}$. Similarly, because $\delta \mathbf{w} \times \mathbf{n}=0$ on $\Gamma_{D}$, we obtain $\mathbf{q}=0$ on $\Gamma_{N}$. Using this last result in (15), we obtain the following boundary condition, $\delta \phi=0$ on $\Gamma_{D}$. Moreover, taking the divergence of the curl equation gives $\Delta \phi=0$ because from (17) we have $\nabla \cdot \mathbf{g}=\nabla \cdot \mathbf{q} / \beta=0$. Hence we obtain $\phi=0$ in $\Omega$. Also, we have the following optimality condition corresponding to (16)

$$
\nabla \times \mathbf{q}-\nabla p+\mathbf{w}-\mathbf{W}=0 \quad \text { in } \Omega .
$$

Now, let us consider the two-dimensional case. We have that the first two components of $\mathbf{q}=\left(q_{1}, q_{2}, q_{3}\right)$ are differentiated with respect to $z$ and therefore they do not appear in (18). However, from (17) we have $\operatorname{div}\left(q_{1}, q_{2}\right)=0$, which is satisfied by $q_{1}=0$ and $q_{2}=0$. For this reason and for notational convenience, we take $\mathbf{q}=(0,0,-q)$. Therefore, using this notation and (16) it follows that

$$
\begin{aligned}
& \mathbf{w}-\mathbf{W}, \delta \mathbf{w})+(\nabla \times \mathbf{q}, \delta \mathbf{w})-(\nabla p, \delta \mathbf{w}) \\
= & (u-U, \delta u)+(v-V, \delta v)-\int_{\Omega} \delta u \frac{\partial q}{\partial y} d x+\int_{\Omega} \delta v \frac{\partial q}{\partial x} d x-\int_{\Omega} \delta u \frac{\partial p}{\partial x} d x-\int_{\Omega} \delta v \frac{\partial p}{\partial y} d x .
\end{aligned}
$$

Hence we can write the adjoint div-curl system in two dimensions as follows

$$
\begin{aligned}
\partial_{x} p+\partial_{y} q & =(u-U) \text { in } \Omega \\
\partial_{x} q-\partial_{y} p & =-(v-V) \text { in } \Omega,
\end{aligned}
$$

with boundary conditions

$$
\begin{aligned}
& p=0 \text { on } \Gamma_{D} \\
& q=0 \text { on } \Gamma_{N} .
\end{aligned}
$$

Summarizing, the solution to (9)-(13) is characterized as the following CR optimality system

$$
\begin{aligned}
\partial_{x} u+\partial_{y} v & =f & & \text { in } \Omega \\
\partial_{x} v-\partial_{y} u & =g & & \text { in } \Omega, \quad \text { (forward CR system) } \\
u n_{y}-v n_{x} & =0 & & \text { on } \Gamma_{D} \\
u n_{x}+v n_{y} & =0 & & \text { on } \Gamma_{N}, \\
\partial_{x} p+\partial_{y} q & =(u-U) & & \text { in } \Omega \\
\partial_{x} q-\partial_{y} p & =-(v-V) & & \text { in } \Omega, \quad \text { (adjoint CR system) } \\
p & =0 & & \text { on } \Gamma_{D} \\
q & =0 & & \text { on } \Gamma_{N}, \\
(\alpha f-p, \tilde{f}-f) & \geq 0 & & \\
(\beta g+q, \tilde{g}-g) & \geq 0 & & \text { for all }(\tilde{f}, \tilde{g}) \in \mathbf{B}_{a d} . \text { (optimality conditions) }
\end{aligned}
$$

Next, we prove that the solution to the optimality system solves the CR optimal control problem. 
Theorem 1 Suppose that there exist a unique solution to (10)-(13) and define $\hat{J}(\mathbf{b}):=J(\mathbf{w}(\mathbf{b}), \mathbf{b})$. Then the constrained CR optimal control problem (9)-(13) has a unique solution $(f, g)$ in $\mathbf{B}_{a d}$ if and only if $(\alpha f-p, \tilde{f}-f)_{L^{2}(\Omega)} \geq 0$ and $(\beta g+q, \tilde{g}-g)_{L^{2}(\Omega)} \geq 0$ for all $(\tilde{f}, \tilde{g}) \in \mathbf{B}_{a d}$, where $p$ and $q$ are the solution to the adjoint CR system (19)-(22).

Proof. Since the forward Cauchy-Riemann problem (10)-(13) has a unique solution $\mathbf{w}=(u, v)$ for a given $\mathbf{b}=(f, g)$, we denote this dependence by $\mathbf{w}=\mathbf{w}(\mathbf{b})$. Therefore, to discuss existence and characterization of the unique solution to (9)(13), we use the reduced cost functional $\hat{J}(\mathbf{b})=J(\mathbf{w}(\mathbf{b}), \mathbf{b})$. That is, we have reduced the PDE constrained optimization problem to a unconstrained optimization problem formulated in the space of the controls.

Recall that the map $\mathbf{L}^{2}(\Omega) \ni \mathbf{b} \mapsto \mathbf{w}(\mathbf{b}) \in \mathbf{H}^{1}(\Omega)$ is affine, continuous, and Fréchet differentiable. Let $\mathbf{w}^{\prime}(\mathbf{b})\left(\mathbf{b}-\mathbf{b}^{*}\right)$ denote its first derivative of the state at $\mathbf{b}=(f, g)$ in the direction of $\left(\mathbf{b}-\mathbf{b}^{*}\right)=\left(f-f^{*}, g-g^{*}\right)$. It is characterized as the solution to

$$
\begin{aligned}
\operatorname{div}\left(\mathbf{w}^{\prime}(\mathbf{b})\left(\mathbf{b}-\mathbf{b}^{*}\right)\right) & =f-f^{*} \\
\operatorname{curl}\left(\mathbf{w}^{\prime}(\mathbf{b})\left(\mathbf{b}-\mathbf{b}^{*}\right)\right) & =g-g^{*},
\end{aligned}
$$

subject to mixed boundary conditions. The second derivative of $\mathbf{b} \mapsto \mathbf{w}(\mathbf{b})$ is zero because of the linear dependence of the governing CR model on the controls.

Next, from (9) we find for the second derivative of $\mathbf{b} \rightarrow \hat{J}(\mathbf{b})$ the following

$$
\hat{J}^{\prime \prime}(\mathbf{b})(\delta \mathbf{b}, \delta \mathbf{b})=\left\|\mathbf{w}^{\prime}(\mathbf{b}) \delta \mathbf{b}\right\|_{\mathbf{L}^{2}(\Omega)}^{2}+\alpha\|\delta f\|_{L^{2}(\Omega)}^{2}+\beta\|\delta g\|_{L^{2}(\Omega)}^{2},
$$

and hence $\mathbf{b} \rightarrow \hat{J}(\mathbf{b})$ is uniformly convex. This implies existence of a unique solution to the optimization problem, denoted by $\mathbf{b}^{*} \in \mathbf{B}_{a d}$. The optimal solution to (9)-(13) is characterized by $\hat{J}^{\prime}(\mathbf{b})\left(\mathbf{b}^{*}, \delta \mathbf{b}\right) \geq 0$ and consequently

$$
\begin{aligned}
\hat{J}^{\prime}\left(\mathbf{b}^{*}\right)\left(\mathbf{b}-\mathbf{b}^{*}\right) & =\left(\mathbf{w}^{*}-\mathbf{W}, \mathbf{w}^{\prime}\left(\mathbf{b}-\mathbf{b}^{*}\right)\right)_{\mathbf{L}^{2}(\Omega)}+\alpha\left(f^{*}, f-f^{*}\right)_{L^{2}(\Omega)}+\beta\left(g^{*}, g-g^{*}\right)_{L^{2}(\Omega)} \\
& \geq 0, \text { for all } \mathbf{b}=(f, g) \in \mathbf{B}_{a d}
\end{aligned}
$$

where $\mathbf{w}^{*}=\mathbf{w}\left(\mathbf{b}^{*}\right)$. To remove $\mathbf{w}^{\prime}$ from this equation, we introduce the Lagrange variables $\left(p^{*}, q^{*}\right) \in \mathbf{H}^{1}(\Omega)$ as the unique solution to the adjoint system (19)-(22). Since

$$
\begin{aligned}
\partial_{x} p^{*}+\partial_{y} q^{*} & =\left(u^{*}-U\right) \\
\partial_{x} q^{*}-\partial_{y} p^{*} & =-\left(v^{*}-V\right),
\end{aligned}
$$

and $\mathbf{w}^{*}-\mathbf{W}=\left(\left(u^{*}-U\right),\left(v^{*}-V\right)\right)$. Using this and (23)-(24) with the Gauss-Green theorem $[6]$ in $(25)$, we obtain

$$
\begin{aligned}
\hat{J}^{\prime}\left(\mathbf{b}^{*}\right)\left(\mathbf{b}-\mathbf{b}^{*}\right) & =-\left(p^{*}, f-f^{*}\right)_{L^{2}(\Omega)}+\alpha\left(f^{*}, f-f^{*}\right)_{L^{2}(\Omega)}+\left(q^{*}, g-g^{*}\right)_{L^{2}(\Omega)} \\
& +\beta\left(g^{*}, g-g^{*}\right)_{L^{2}(\Omega)} \\
& =\left(\alpha f^{*}-p^{*}, f-f^{*}\right)_{L^{2}(\Omega)}+\left(\beta g^{*}+q^{*}, g-g^{*}\right)_{L^{2}(\Omega)} \\
& \geq 0 \text { for all }(f, g) \in \mathbf{B}_{a d}
\end{aligned}
$$

Hence, we obtain the variational inequalities $(\alpha f-p, \tilde{f}-f)_{L^{2}(\Omega)} \geq 0$ and $(\beta g+q, \tilde{g}-$ $g)_{L^{2}(\Omega)} \geq 0$ for all $(\tilde{f}, \tilde{g}) \in \mathbf{B}_{a d}$, and $\hat{J}^{\prime}\left(\mathbf{b}^{*}\right)\left(\mathbf{b}-\mathbf{b}^{*}\right) \geq 0$ that constitute the necessary and sufficient optimality conditions for the constrained CR optimal control problem. 


\section{Discretization of constrained CR optimality system}

In this section, we discuss the discretization of the constrained CR optimality system by finite-difference approximations on staggered grids [2]. This discretization framework is typical in the approximation of first-order elliptic systems [9].

Consider a sequence of grids $\left\{\Omega_{h}\right\}_{h>0}$ defined by

$$
\Omega_{h}=\left\{\mathbf{x} \in \mathbb{R}^{2}: x_{i}=s_{i} h_{x}, y_{j}=s_{j} h_{y}, \quad s_{i}, s_{j} \in \mathbb{Z}\right\} \cap \Omega .
$$

We assume that $\Omega$ is a rectangular domain and that the values of $h_{x}$ and $h_{y}$ are chosen such that the boundaries of $\Omega$ coincide with grid lines. On staggered grids, variables may be placed on cell vertices, cell centers, and cell edges that can be horizontal and vertical. We denote these sets of grid points with $\Omega_{h}^{s}, s \in\{v, c, e h, e v\}$ with the obvious meaning of the alphabetic index. Notice that within the same set the grid points are spaced with $h_{x}$ and $h_{y}$ intervals in the $x$ - and $y$-direction, respectively.

For grid functions $u^{h}$ and $v^{h}$ defined on the same set $\Omega_{h}^{s}$, we introduce the discrete $L^{2}$-scalar product

$$
\left(u^{h}, v^{h}\right)_{L_{h}^{2}\left(\Omega_{h}^{s}\right)}=h_{x} h_{y} \sum_{\mathbf{x} \in \Omega_{h}^{s}} u^{h}(\mathbf{x}) v^{h}(\mathbf{x})
$$

with associated norm $\left\|u^{h}\right\|_{L_{h}^{2}\left(\Omega_{h}^{s}\right)}=\left(u^{h}, u^{h}\right)_{L_{h}^{2}\left(\Omega_{h}^{s}\right)}^{1 / 2}$. The spaces $L_{h}^{2}\left(\Omega_{h}^{s}\right)$ consist of the sets of grid functions $u^{h}$ defined on $\Omega_{h}^{s}$ endowed with $\left\|u^{h}\right\|_{L_{h}^{2}\left(\Omega_{h}^{s}\right)}$ as norm.

In the following, we denote with $\mathcal{U}_{h}, \mathcal{V}_{h}, \mathcal{P}_{h}$, and $\mathcal{Q}_{h}$ the space of the grid functions $u^{h}, v^{h}, p^{h}$, and $q^{h}$, that approximate the state and adjoint variables, respectively.

First, we discuss the discretization of the CR state model. In the staggered grid the variable $u$ is defined on $\Omega_{h}^{e v}$ while $v$ is defined on $\Omega_{h}^{e h}$.

We consider a unique set of grid indices $(i, j), i=1, \ldots, N_{x}+1, j=1, \ldots, N_{y}$ that index all grid points including the boundaries in a lexicographic order starting from the lowest-left corner $i=1, j=1$. The vertices coordinate are given by $x_{i}=(i-1) h_{x}$ and $y_{j}=(j-1) h_{y}$. With $v_{i+1 / 2, j+1 / 2}$ we mean the discrete counterpart to $v\left(x_{i}+h_{x} / 2, y_{j}+h_{y} / 2\right)$. The set of forward CR equations is given by

$$
\begin{array}{ll}
\frac{u_{i+1, j}-u_{i, j}}{h_{x}}+\frac{v_{i+1 / 2, j+1 / 2}-v_{i+1 / 2, j-1 / 2}}{h_{y}}=f_{i+1 / 2, j}, & \text { on } \Omega_{h}^{c} \\
\frac{v_{i+1 / 2, j+1 / 2}-v_{i-1 / 2, j+1 / 2}}{h_{x}}-\frac{u_{i, j+1}-u_{i, j}}{h_{y}}=g_{i, j+1 / 2}, & \text { on } \Omega_{h}^{v}
\end{array}
$$

where the divergence equation (27) is centered at all inner cell centers and the curl equation is centered at all internal cell vertices. With this discretization, secondorder accurate solutions are obtained [7].

Next, we discretize the adjoint CR system as follows

$$
\begin{aligned}
\frac{p_{i+1 / 2, j}-p_{i-1 / 2, j}}{h_{x}}+\frac{q_{i, j+1 / 2}-q_{i, j-1 / 2}}{h_{y}} & =(u-U)_{i, j}, \quad \text { on } \Omega_{h}^{e v} \\
\frac{q_{i+1, j+1 / 2}-q_{i, j+1 / 2}}{h_{x}}-\frac{p_{i+1 / 2, j+1}-p_{i+1 / 2, j}}{h_{y}} & \left.=-(v-V)_{i+1 / 2, j+1 / 2}, \quad \text { on } \Omega_{i}^{\text {qb }} 30\right)
\end{aligned}
$$

where the adjoint div equation (29) is centered at all internal vertical cell edges and the adjoint curl equation (30) is centered at all internal horizontal cell edges. 
We notice that the optimality conditions $(\alpha f-p, \tilde{f}-f) \geq 0$ and $(\beta g+q, \tilde{g}-g) \geq 0$ suggest that the staggered grid for the adjoint CR system should be such that $p$, similarly to $f$, is defined on $\Omega_{h}^{c}$, and $q$, similarly to $g$, is defined on $\Omega_{h}^{v}$. In this way, we implement a direct coupling between state, adjoint, and control variables without the need of interpolation.

In Figure 1, we depict the staggered grid for the optimality system, including the allocation of all variables. Notice that with our approach based on the optimality conditions, the variables $f, p$, and the variables $g, q$, share the same location and as a by-product they result appropriately located on the boundaries in order to implement the boundary conditions.

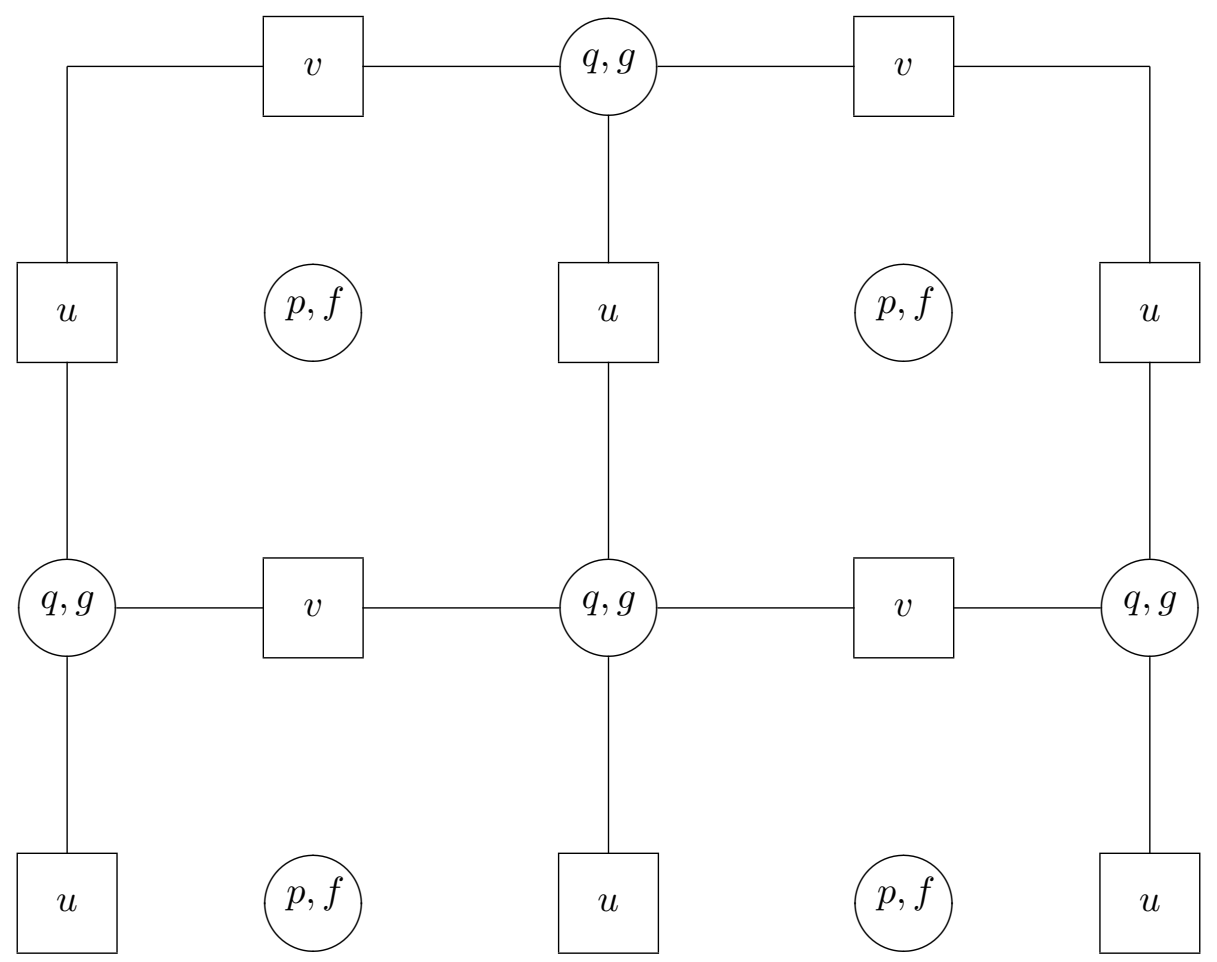

Figure 1: Staggered grid for the CR optimality system.

Summarizing, the discrete control-constrained Cauchy-Riemann optimality system results as follows

$$
\begin{aligned}
\frac{u_{i+1, j}-u_{i, j}}{h_{x}}+\frac{v_{i+1 / 2, j+1 / 2}-v_{i+1 / 2, j-1 / 2}}{h_{y}} & =f_{i+1 / 2, j}, & & \text { on } \Omega_{h}^{c} \\
\frac{v_{i+1 / 2, j+1 / 2}-v_{i-1 / 2, j+1 / 2}}{h_{x}}-\frac{u_{i, j+1}-u_{i, j}}{h_{y}} & =g_{i, j+1 / 2}, & & \text { on } \Omega_{h}^{v} \\
\frac{p_{i+1 / 2, j}-p_{i-1 / 2, j}}{h_{x}}+\frac{q_{i, j+1 / 2}-q_{i, j-1 / 2}}{h_{y}} & =(u-U)_{i, j}, & & \text { on } \Omega_{h}^{e v} \\
\frac{q_{i+1, j+1 / 2}-q_{i, j+1 / 2}}{h_{x}}-\frac{p_{i+1 / 2, j+1}-p_{i+1 / 2, j}}{h_{y}} & =-(v-V)_{i+1 / 2, j+1 / 2}, & & \text { on } \Omega_{h}^{e h} \\
\left(\alpha f_{i+1 / 2, j}-p_{i+1 / 2, j}, \tilde{f}_{i+1 / 2, j}-f_{i+1 / 2, j}\right) & \geq 0, & & \text { in } \Omega_{h}^{c} \\
\left(\beta g_{i, j+1 / 2}+q_{i, j+1 / 2}, \tilde{g}_{i, j+1 / 2}-g_{i, j+1 / 2}\right) & \geq 0, & & \text { in } \Omega_{h}^{v}
\end{aligned}
$$


for all $\left(\tilde{f}_{i+1 / 2, j}, \tilde{g}_{i, j+1 / 2}\right) \in \mathbf{B}_{a d h}$, where $\mathbf{B}_{a d h}$ is the discrete analog of $\mathbf{B}_{a d}$; with the following boundary conditions

$$
\begin{array}{rll}
u_{i, j}=0 & \text { for } i=1, \ldots, N_{x}+1, j=1 ; i=1, N_{x}+1, j=1, \ldots, N_{y} \\
v_{i+1 / 2, j+1 / 2}=0 & \text { for } i=1, \ldots, N_{x}, j=N_{y} \\
p_{i+1 / 2, j}=0 & \text { for } i=1, \ldots, N_{x}, j=1 \\
q_{i, j+1 / 2}=0 & \text { for } i=2, \ldots, N_{x}, j=N_{y} ; i=1, N_{x}+1, j=1, \ldots, N_{y}-1 .
\end{array}
$$

\section{$5 \quad$ A full multigrid framework}

We develop a full multigrid scheme to solve the discrete optimality system (31)-(32) defined on staggered grids as the one depicted in Figure 1 (coarsest grid). Our work extends previous developments on multigrid methods for CR systems $[4,2,13]$ to the case of control-constrained CR optimality systems. This development faces some difficulties due to the nature of the staggered grids and of the structure of the coupled forward and adjoint CR equations.

To illustrate our approach, we discuss the full approximation scheme (FAS) and the full multigrid method (FMG). The FAS scheme is a nonlinear multigrid method that allows to accommodate nonlinearities like the inequalities representing the optimality conditions. The FMG scheme allows to improve the computational complexity of the FAS scheme. The FMG scheme is obtained combining a nested iteration strategy with the FAS scheme that we discuss next.

Consider the optimality system (31)-(32) at the discretization level $k$ for the unknown variables $\Phi_{k}=\left(u_{k}, v_{k}, p_{k}, q_{k}, f_{k}, g_{k}\right)$. We write this system in a compact notation as the following nonlinear problem

$$
A_{k}\left(\Phi_{k}\right)=F_{k} .
$$

To solve this problem, the FAS scheme combines two complementary solution strategies to solve efficiently for the short- and long-scale error components of the optimal solution. For the short-scale components, a smoothing algorithm is introduced, while the long-scale error components should be solved by a coarse-grid correction strategy.

We denote with $\Phi_{k}^{(l)}=S_{k}\left(\Phi_{k}^{(l-1)}, F_{k}\right)$, the result of our smoothing scheme given below in Section 5.1. Suppose to apply $m_{1}$-times this iteration to (33) starting with the current approximation $\Phi_{k}^{(0)}$ to obtain the approximate solution $\tilde{\Phi}_{k}=\Phi_{k}^{\left(m_{1}\right)}$; see $[1,3]$. Denote with $e_{k}^{\left(m_{1}\right)}=\Phi_{k}-\Phi_{k}^{\left(m_{1}\right)}$ the solution error after smoothing. This function should be a smoother function than $e_{k}^{(0)}=\Phi_{k}-\Phi_{k}^{(0)}$.

It is clear that in a nonlinear setting the desired correction $e_{k}$ to $\tilde{\Phi}_{k}$ is defined by $A_{k}\left(\tilde{\Phi}_{k}+e_{k}\right)=F_{k}$. This correction can be defined as the solution to

$$
A_{k}\left(\tilde{\Phi}_{k}+e_{k}\right)-A_{k}\left(\tilde{\Phi}_{k}\right)=r_{k},
$$

where $r_{k}=F_{k}-A_{k}\left(\tilde{\Phi}_{k}\right)$ is the residual associated to $\tilde{\Phi}_{k}$.

Now, consider to represent the problem (34) on the coarser grid $\Omega_{k-1}$ with the help of $I_{k}^{k-1}$ and $I_{k-1}^{k}$, a restriction and prolongation operator, respectively. To represent $\tilde{\Phi}_{k}+e_{k}$ on this coarse grid we write

$$
\Phi_{k-1}:=I_{k}^{k-1} \tilde{\Phi}_{k}+e_{k-1} .
$$


Since $I_{k}^{k-1} \Phi_{k}$ and $\tilde{\Phi}_{k}$ should represent the same function but on different grids. We can think of representing $e_{k}$ by a coarse function $e_{k-1}$ because $e_{k}$ is smooth due to the action of $S_{k}$.

Now to formulate (34) on the coarse grid replace $A_{k}(\cdot)$ by $A_{k-1}(\cdot), \tilde{\Phi}_{k}$ by $I_{k}^{k-1} \tilde{\Phi}_{k}$, and $r_{k}$ by restriction $I_{k}^{k-1} r_{k}=I_{k}^{k-1}\left(F_{k}-A_{k}\left(\tilde{\Phi}_{k}\right)\right)$. We get the following (FAS) equation

$$
A_{k-1}\left(\Phi_{k-1}\right)=I_{k}^{k-1}\left(F_{k}-A_{k}\left(\tilde{\Phi}_{k}\right)\right)+A_{k-1}\left(I_{k}^{k-1} \tilde{\Phi}_{k}\right) .
$$

This equation is also written in the form $A_{k-1}\left(\Phi_{k-1}\right)=I_{k}^{k-1} F_{k}+\tau_{k}^{k-1}$ where

$$
\tau_{k}^{k-1}=A_{k-1}\left(I_{k}^{k-1} \tilde{\Phi}_{k}\right)-I_{k}^{k-1} A_{k}\left(\tilde{\Phi}_{k}\right) .
$$

The term $\tau_{k}^{k-1}$ is the fine-to-coarse defect or residual correction such that at convergence the solution to (36) coincides with the fine grid solution in the sense that $\Phi_{k-1}=I_{k}^{k-1} \Phi_{k}$. With $\Phi_{k-1}$ obtained solving (36) and from (35) we have

$$
e_{k-1}=\Phi_{k-1}-I_{k}^{k-1} \tilde{\Phi}_{k}
$$

Therefore we can obtain a coarse-grid correction to the fine-grid approximation as follows

$$
\Phi_{k}=\tilde{\Phi}_{k}+I_{k-1}^{k}\left(\Phi_{k-1}-I_{k}^{k-1} \tilde{\Phi}_{k}\right) .
$$

where $I_{k-1}^{k}$ is the coarse-to-fine interpolation operator. To damp possible highfrequency errors arising through the entire coarse-grid correction process, the correction step (37) is followed by $m_{2}$-times smoothing iteration.

The FAS cycle is summarized in the following algorithm.

Algorithm 2 FAS $\left(m_{1}, m_{2}\right)$ for solving $A_{k}\left(\Phi_{k}\right)=F_{k}$.

1. If $k=1$ solve $A_{k}\left(\Phi_{k}\right)=F_{k}$ exactly.

2. Pre-smoothing steps on the fine grid: $\Phi_{k}^{(l)}=S_{k}\left(\Phi_{k}^{(l-1)}, F_{k}\right), l=1, \ldots, m_{1}$;

3. Computation of the residual: $r_{k}=F_{k}-A_{k}\left(\Phi_{k}^{\left(m_{1}\right)}\right)$;

4. Restriction of the residual: $r_{k-1}=I_{k}^{k-1} r_{k}$;

5. Set $\Phi_{k-1}=I_{k}^{k-1} \Phi_{k}^{\left(m_{1}\right)}$;

6. Set $F_{k-1}=r_{k-1}+A_{k-1}\left(\Phi_{k-1}\right)$

7. Call $m$ times $F A S\left(m_{1}, m_{2}\right)$ to solve $A_{k-1}\left(\Phi_{k-1}\right)=F_{k-1}$;

8. Coarse-grid correction: $\Phi_{k}^{\left(m_{1}+1\right)}=\Phi_{k}^{\left(m_{1}\right)}+I_{k-1}^{k}\left(\Phi_{k-1}-I_{k}^{k-1} \Phi_{k}^{\left(m_{1}\right)}\right)$;

9. Post-smoothing steps on the fine grid: $\Phi_{k}^{(l)}=S_{k}\left(\Phi_{k}^{(l-1)}, F_{k}\right), l=m_{1}+2, \ldots, m_{1}+$ $m_{2}+1$;

Notice that we can perform $m$ two-grid iterations at each working level. For $m=1$ we have a $V$-cycle and for $m=2$ we have a $W$-cycle; $m$ is called the cycle index [16].

When dealing with nonlinear problems it is essential to start the iterative procedure from a good initial approximation. The multigrid setting suggests a natural 
way of how to get this approximation. Suppose to start the solution process from a coarse working level $K<M$ where the discretized problem $A_{l}\left(\Phi_{l}\right)=F_{l}$ with $l=K$ is easily solved. The idea is to interpolate this solution to the next finer working level as initial approximation for the iterative process to solve $A_{l+1}\left(\Phi_{l+1}\right)=F_{l+1}$ as $\Phi_{l+1}=I_{l}^{l+1} \Phi_{l}$. Thereafter the FAS solution process at level $l+1$ is applied. The idea of using a coarse grid approximation as a first guess for the solution process on a finer grid is known as nested iteration. The algorithm obtained by combining the multigrid scheme with nested iteration is called full multigrid (FMG) method. Because of the improvement on the initial solution at each starting level, the FMG scheme results to be more efficient than the iterative application of the multigrid cycle without FMG initialization. A description of the FMG scheme is given in Figure 2 and in the following algorithm.

Algorithm 3 FMG scheme for solving $A_{L}\left(\Phi_{L}\right)=F_{L}$.

1. For $l=K<L$ set initial approximation $u_{l}$.

2. If $l<L$ then interpolate to the next finer working level: $\tilde{\Phi}_{l+1}=I_{l}^{l+1} \Phi_{l}$;

3. Apply FAS to solve $A_{l+1}\left(\Phi_{l+1}\right)=F_{l+1}$, starting with $\tilde{\Phi}_{l+1}$;

4. Set $l:=l+1$. If $l<L$ go to 2; else stop.

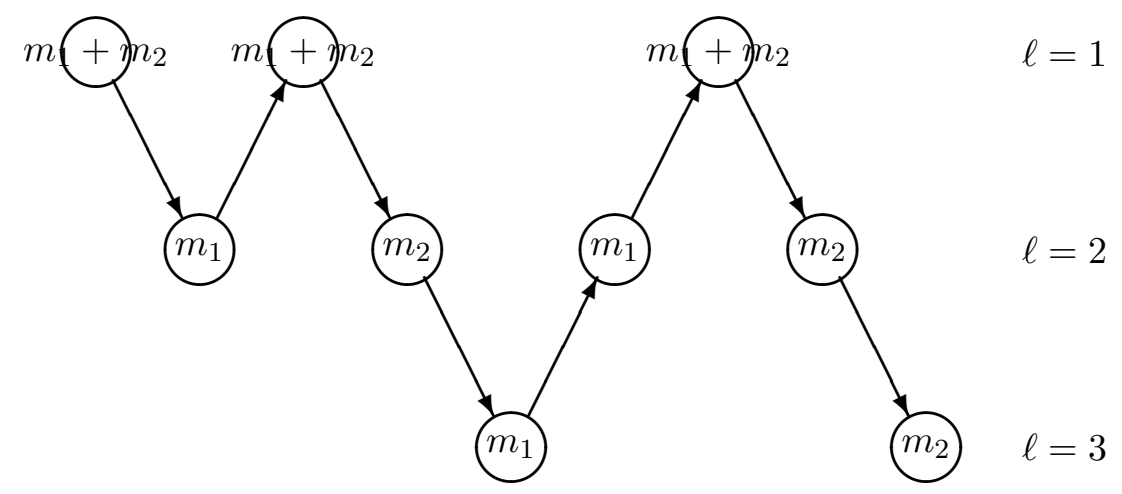

Figure 2: The FMG scheme.

Concerning the hierarchy of grids, they are obtained by refining a starting coarse grid; see Figure 1. This refinement process usually consists in halving the mesh size in some or all directions [16]. However, in the case of staggered grids this procedure results in a non-nested hierarchy of grids such that the same type of variables are placed on different spatial coordinates corresponding to different levels of the hierarchy. This fact is well known and requires additional effort in the construction of the required intergrid transfer operators; see $[2,13]$. To alleviate these difficulties and recover the advantages of a nested sequence of grids, we notice that starting from the given coarse grid a nested sequence of grids is obtained by tripling the 
mesh size. This remark seems novel in the staggered-grids context and it has important consequences in the development of multigrid schemes on staggered grids. See [5] for a coarsening strategy by a factor of three in the context of cell-centered discretizations of second-order PDE systems.

In the following, we illustrate our smoothing scheme and the other components of our full multigrid method. We define a sequence of nested grids (or levels) $\Omega_{k}$ of mesh size $h_{x k}=h_{x 1} / 3^{(k-1)}$ and $h_{y_{k}}=h_{y_{1}} / 3^{(k-1)}, k=1, \ldots, L$, where $k=L$ is the finest level and $h_{x 1}$ and $h_{y_{1}}$ are the mesh sizes of the coarsest grid in the $x$ and $y$ direction, respectively. In the following, denote all operators and functions defined on $\Omega_{k}$ in terms of the index $k$.

\subsection{A smoothing scheme and intergrid transfer operators}

In this section, we discuss a smoothing schemes for the CR optimality system and the prolongation and restriction operators required for the development of the coarsegrid correction step.

The construction of smoothing schemes is commonly based on a one-to-one correspondence between equations and unknowns on grid points where a step of the smoothing iteration is defined with the purpose to satisfy the algebraic equation associated to the variables defined on the given grid point. Such one-to-one correspondence is natural in second-order elliptic problems but not on first-order systems. For this reason distributive relaxation [2] was proposed that aims at satisfying at each step the set of discrete equations by distributing changes to several unknowns.

Let $\left(u^{h}, v^{h}, f h, g^{h}\right)$ be the current approximation to the numerical solution. We define an update to this approximation by an iterative step.

First, we update the controls $f^{h}$ and $g^{h}$. For this purpose, we perform a gradient update which includes projection on the control constraints as follows

$$
\begin{aligned}
f^{*, h} & :=\mathbb{P}_{[\underline{f}, \bar{f}]}\left(f^{h}-t\left(\alpha f^{h}-p^{h}\right)\right) \\
g^{*, h} & :=\mathbb{P}_{[\underline{g, \bar{g}]}}\left(g^{h}-t\left(\beta g^{h}+q^{h}\right)\right)
\end{aligned}
$$

where $\nabla_{f} \hat{J}\left(f^{h}, g^{h}\right)=\alpha f^{h}-p^{h}, \nabla_{g} \hat{J}\left(f^{h}, g^{h}\right)=\beta g^{h}+q^{h}$, and the steplength $t \in(0,1]$. The projection $\mathbb{P}_{[f, \bar{f}]}$ is given by

$$
\mathbb{P}_{[\underline{f}, \bar{f}]}(f)= \begin{cases}\bar{f} & \text { if } f>\bar{f} \\ f & \text { if } f \leq f \leq \bar{f} \\ \underline{f} & \text { if } \bar{f}<\underline{f}\end{cases}
$$

Corresponding to the new values of the controls, we update the state and adjoint variables. For this purpose, we consider the divergence equation defined at the cell center $(i+1 / 2, j), i=1, \ldots, N_{x}, j=2, \ldots, N_{y}$, where we compute the following 'dynamic' residual [2]. We have

$$
r_{1}^{h}=f^{h}-\partial_{x}^{h} u^{h}-\partial_{y}^{h} v^{h} .
$$

We call $r_{1}$ a dynamic residual because it is the residual at the cell center just before 
the update step. This step is as follows

$$
\begin{aligned}
u_{i+1, j} & \leftarrow u_{i+1, j}+h_{x} h_{y}^{2} \delta_{1} \\
u_{i, j} & \leftarrow u_{i, j}-h_{x} h_{y}^{2} \delta_{1} \\
v_{i+1 / 2, j+1 / 2} & \leftarrow v_{i+1 / 2, j+1 / 2}+h_{y} h_{x}^{2} \delta_{1} \\
v_{i+1 / 2, j-1 / 2} & \leftarrow v_{i+1 / 2, j-1 / 2}-h_{y} h_{x}^{2} \delta_{1}
\end{aligned}
$$

where

$$
\delta_{1}=\frac{1}{2\left(h_{x}^{2}+h_{y}^{2}\right)} r_{1}^{h} .
$$

With this distributed update, we have that the residual (38) results zero while the residuals of the curl equation at all vertices remain unchanged. In the case where one of the updates at the boundaries are not allowed because of boundary conditions, we need to modify $\delta_{1}$ as follows

$$
\delta_{1}=\frac{1}{2\left(h_{x}^{2}+h_{y}^{2}\right)-d} r_{1}^{h}
$$

where $d \in\left\{h_{x}^{2}, h_{y}^{2}, h_{x}^{2}+h_{y}^{2}\right\}$ depending upon which boundary is touched. That is, $d=h_{y}^{2}$ whenever one of the $u$ updates is not performed and $d=h_{x}^{2}$ if one of the $v$ updates is not performed. We have $d=h_{x}^{2}+h_{y}^{2}$ in the case when both $u$ and $v$ are not updated at the boundary (corner).

The update step for the curl equation at $(i, j+1 / 2), i=2, \ldots, N_{x}, j=1, \ldots, N_{y}-$ 1 , is made in a similar way. Consider the dynamic residual

$$
r_{2}^{h}=g^{h}+\partial_{y}^{h} u^{h}-\partial_{x}^{h} v^{h},
$$

at $(i, j+1 / 2)$ before the update. Define

$$
\delta_{2}=\frac{1}{2\left(h_{x}^{2}+h_{y}^{2}\right)} r_{2}^{h} .
$$

The update step for the curl equation is given by

$$
\begin{aligned}
u_{i, j+1} & \leftarrow u_{i, j+1}-h_{y} h_{x}^{2} \delta_{2} \\
u_{i, j} & \leftarrow u_{i, j}+h_{y} h_{x}^{2} \delta_{2} \\
v_{i+1 / 2, j+1 / 2} & \leftarrow v_{i+1 / 2, j+1 / 2}+h_{x} h_{y}^{2} \delta_{2} \\
v_{i-1 / 2, j+1 / 2} & \leftarrow v_{i-1 / 2, j+1 / 2}-h_{x} h_{y}^{2} \delta_{2}
\end{aligned}
$$

This distributed update is such that the residuals of the divergence equation at all centers remains unchanged while the residual $(39)$ at $(x, y)$ becomes zero. Also in the relaxation of the curl equation, the term $\delta_{2}$ becomes

$$
\delta_{2}=\frac{1}{2\left(h_{x}^{2}+h_{y}^{2}\right)-d} r_{2}^{h},
$$

where $d \in\left\{h_{x}^{2}, h_{y}^{2}, h_{x}^{2}+h_{y}^{2}\right\}$ depending upon which boundary is touched. That is, $d=h_{x}^{2}$ whenever one of the $u$ updates is not performed and $d=h_{y}^{2}$ if one of the $v$ updates is not performed. We have $d=h_{x}^{2}+h_{y}^{2}$ in the case when both $u$ and $v$ are not updated at the boundary. 
Next, we discuss the distributed relaxation for the adjoint system. We consider the adjoint divergence equation defined at $(i, j), i=2, \ldots, N_{x}, j=2, \ldots, N_{y}$, where the following 'dynamic' residual is computed

$$
r_{3}^{h}=(u-U)^{h}-\partial_{x}^{h} p^{h}-\partial_{y}^{h} q^{h} .
$$

Based on this dynamic residual, we have the following relaxation step for the first equation of the adjoint system

$$
\begin{aligned}
& p_{i+1 / 2, j} \leftarrow p_{i+1 / 2, j}+h_{x} h_{y}^{2} \delta_{3} \\
& p_{i-1 / 2, j} \leftarrow p_{i-1 / 2, j}-h_{x} h_{y}^{2} \delta_{3} \\
& q_{i, j+1 / 2} \leftarrow q_{i, j+1 / 2}+h_{y} h_{x}^{2} \delta_{3} \\
& q_{i, j-1 / 2} \leftarrow q_{i, j-1 / 2}-h_{y} h_{x}^{2} \delta_{3}
\end{aligned}
$$

where the term $\delta_{3}$ is given by

$$
\delta_{3}=\frac{1}{2\left(h_{x}^{2}+h_{y}^{2}\right)-d} r_{3}^{h},
$$

where $d$ is defined as in the forward divergence equation.

The adjoint curl equation is defined at $(i+1 / 2, j+1 / 2), i=1, \ldots, N_{x}, j=$ $1, \ldots, N_{y}-1$, we compute the corresponding residual as follows

$$
r_{4}^{h}=-(v-V)^{h}-\partial_{x}^{h} q^{h}+\partial_{y}^{h} p^{h} .
$$

The distributed relaxation results in the following update step for the second equation of the adjoint system

$$
\begin{aligned}
p_{i+1 / 2, j+1} & \leftarrow p_{i+1 / 2, j+1}-h_{y} h_{x}^{2} \delta_{4} \\
p_{i+1 / 2, j} & \leftarrow p_{i+1 / 2, j}+h_{y} h_{x}^{2} \delta_{4} \\
q_{i+1, j+1 / 2} & \leftarrow q_{i+1, j+1 / 2}+h_{x} h_{y}^{2} \delta_{4} \\
q_{i, j+1 / 2} & \leftarrow q_{i, j+1 / 2}-h_{x} h_{y}^{2} \delta_{4}
\end{aligned}
$$

where the term $\delta_{4}$ is given by

$$
\delta_{4}=\frac{1}{2\left(h_{x}^{2}+h_{y}^{2}\right)-d} r_{4}^{h},
$$

where $d$ is defined as in the forward curl equation.

The above steps update the forward and adjoint variables for the given set of new controls obtained with the projected-gradient step. Next, we discuss the intergrid transfer operators.

Since the governing equations are only first-order differential equations, it is admissible to have a coarse-to-fine first-order piecewise interpolation [10]. Indeed, one can use higher-order interpolation which results advantageous but that would be difficult to implement in a non-nested staggered grid. On the other hand, using a coarsening of a factor of three, a nested staggered grid is obtained and the implementation of bilinear interpolation results easier.

In order to illustrate the interpolation procedure, consider the space $\mathcal{U}_{k}, k=$ $1, \ldots, L$. Among two grids $\Omega_{k}$ and $\Omega_{k-1}$, we define a prolongation operator, $I_{k-1}^{k}$ : 
$\mathcal{U}_{k-1} \rightarrow \mathcal{U}_{k}$, that is consistent with the assumption of bilinear finite elements on each rectangular partition of the discretization. That is, on each rectangular partition $\left[x_{I}, x_{I+1}\right] \times\left[y_{J}, y_{J+1}\right]$ of $\bar{\Omega}_{k-1}$, define $s_{x}=x_{I+1}-x_{I}$ and $s_{y}=y_{J+1}-y_{J}$, and the piecewise bilinear function which interpolates $u$ at $(x, y)$ is given by

$$
\begin{aligned}
\tilde{u}(x, y) & =\frac{\left(x_{I+1}-x\right)\left(y_{J+1}-y\right)}{s_{x} s_{y}} u_{I J}+\frac{\left(x-x_{I}\right)\left(y_{J+1}-y\right)}{s_{x} s_{y}} u_{I+1} \\
& +\frac{\left(x_{I+1}-x\right)\left(y-y_{J}\right)}{s_{x} s_{y}} u_{I J+1}+\frac{\left(x-x_{I}\right)\left(y-y_{J}\right)}{s_{x} s_{y}} u_{I+1}{ }_{I+1} .
\end{aligned}
$$

Notice that the prolongation of $u$ on nested grid points is the identity.

In order to transfer residuals and solution functions from the fine to the coarse grids, we use the straight injection operator $I_{k}^{k-1}: \mathcal{U}_{k} \rightarrow \mathcal{U}_{k-1}$. The same operator is applied to the other spaces of grid functions.

\section{$6 \quad$ Numerical experiments}

In this section, we present results of numerical experiments with control-constrained Cauchy-Riemann optimal control problems discretized on staggered grids and solved with a full multigrid scheme.

Consider the CR optimal control problem (9) - (13) on the rectangular domain $\Omega=(0,1) \times(0,3 / 2)$, where $\Gamma_{D}=(0,1) \times\{0\}$ and $\Gamma_{N}=\partial \Omega \backslash \bar{\Gamma}_{D}$. For target functions $(U, V)$, we consider a "recirculating flow" as follows

$$
\begin{aligned}
U(x, y) & :=-\sin (\pi x) \cos \left(\frac{2 \pi}{3} y\right) \\
V(x, y) & :=\cos (\pi x) \sin \left(\frac{2 \pi}{3} y\right)
\end{aligned}
$$

The boundary conditions for the forward problem are given by

$$
\begin{array}{llll}
u=0 & \text { on } & \Gamma_{D}=(0,1) \times\{0\} \\
u=0 & \text { on } & \Gamma_{1}=\{0\} \times(0,3 / 2) \\
u=0 & \text { on } & \Gamma_{2}=\{1\} \times(0,3 / 2) \\
v=0 & \text { on } & \Gamma_{3}=(0,1) \times\{3 / 2\}
\end{array}
$$

and for adjoint system we have the following

$$
\begin{array}{llll}
p=0 & \text { on } & \Gamma_{D}=(0,1) \times\{0\} \\
q=0 & \text { on } & \Gamma_{1}=\{0\} \times(0,3 / 2) \\
q=0 & \text { on } & \Gamma_{2}=\{1\} \times(0,3 / 2) \\
q=0 & \text { on } & \Gamma_{3}=(0,1) \times\{3 / 2\} .
\end{array}
$$

To solve the corresponding control problem, we use our full multigrid method applied to the optimality system (31)-(32). We employ $W$-cycles and $m_{1}=m_{2}=2$ and find advantageous to choose $t=1$ for the gradient update of the smoothing procedure. The choice of $W$-cycles is advantageous when the constraints on the control are active because in this case additional effort is required to compute with active sets. 
In the FMG scheme, we implement a stopping criteria with a given tolerance on the discrete $L^{2}$-norm of the residuals as follows

$$
\max \left\{\left\|r_{u}^{h}\right\|_{L^{2}},\left\|r_{v}^{h}\right\|_{L^{2}},\left\|r_{p}^{h}\right\|_{L^{2}},\left\|r_{q}^{h}\right\|_{L^{2}}\right\}<10^{-6} .
$$

We consider two sets of experiments. In the first set, we report results for the unconstrained-control case to show the computational performance of the FMG scheme and the tracking ability of the optimal control formulation without constraints on the control. In the second set of experiments, we introduce constraints on the controls.

For the unconstrained-control case, we depict in Figure 3 the optimal solution with $\alpha=\beta=10^{-1}$ and a fine mesh $N_{x} \times N_{y}=162 \times 122$.
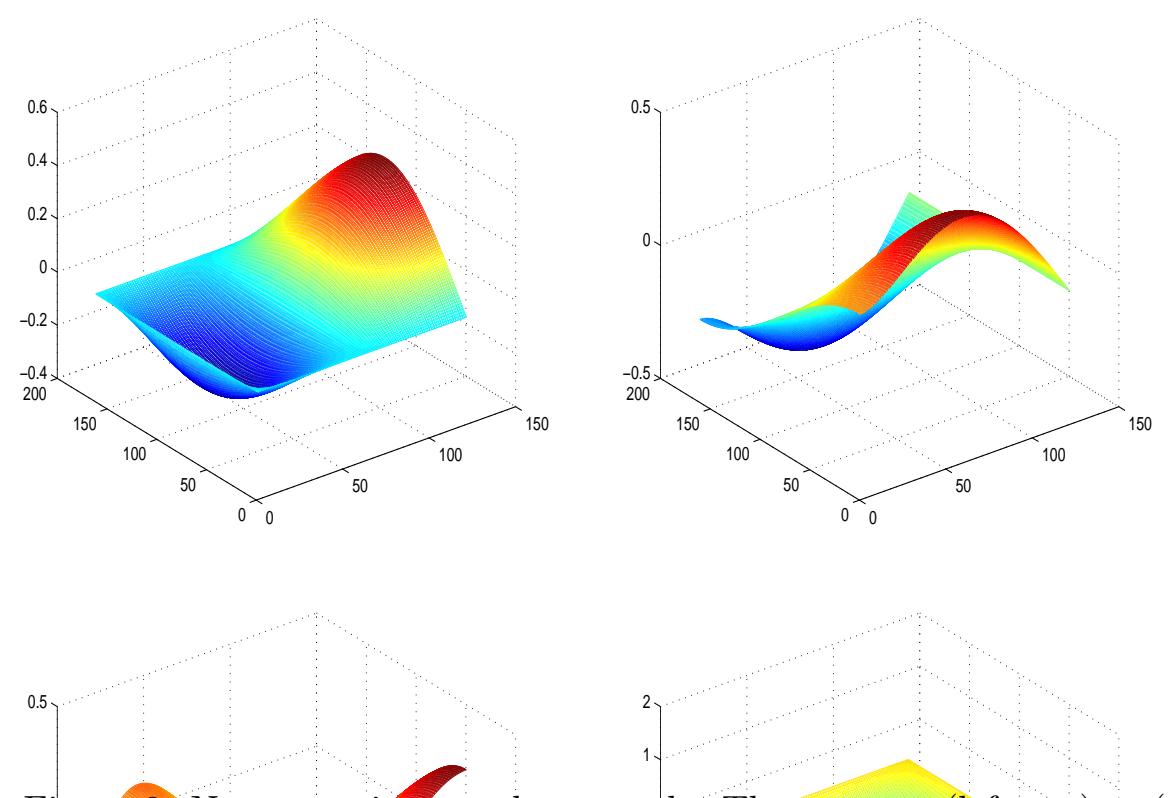

Figure 3: No constraints on the controls. The states $u$ (left top), $v$ (right top) and controls $f$ (left bottom), $g$ (right bettom) with $\alpha=\beta=10^{-1}$ and $N_{x} \times N_{y}=$ $162 \times 122$.

${ }_{200}^{-0.5}$ In Table 1, we report tracking exrors for different values of the optimization parameters, including the case $\alpha \neq \beta .^{150}$ hoo obtain improved ${ }_{100}^{150}$ tracking for smaller values of the contro ${ }^{0}$ weights.

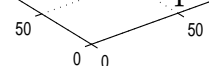

Next, we introduce constraints on the controls as follows. We require $-0.2 \leq$ $f \leq 0.2$ and $-1.75 \leq g \leq 1$, as control constraints such that both control constraints are active for all choices $\alpha, \beta \in\left\{10^{-1}, 10^{-2}, 10^{-3}, 10^{-4}\right\}$; see Figure 4 and Figure 5 . 
Table 1: $L^{2}$-norm of the tracking errors. No constraints on the controls.

\begin{tabular}{lcc}
\hline$\alpha, \beta$ & $\|u-U\|_{L^{2}}$ & $\|u-V\|_{L^{2}}$ \\
\hline \hline & & \\
$10^{-2}, 10^{-2}$ & $2.11 \mathrm{E}-01$ & $7.84 \mathrm{E}-01$ \\
$10^{-2}, 10^{-4}$ & $1.57 \mathrm{E}-01$ & $1.57 \mathrm{E}-02$ \\
$10^{-4}, 10^{-2}$ & $2.04 \mathrm{E}-01$ & $9.33 \mathrm{E}-02$ \\
$10^{-4}, 10^{-4}$ & $1.55 \mathrm{E}-01$ & $1.36 \mathrm{E}-02$ \\
\hline
\end{tabular}
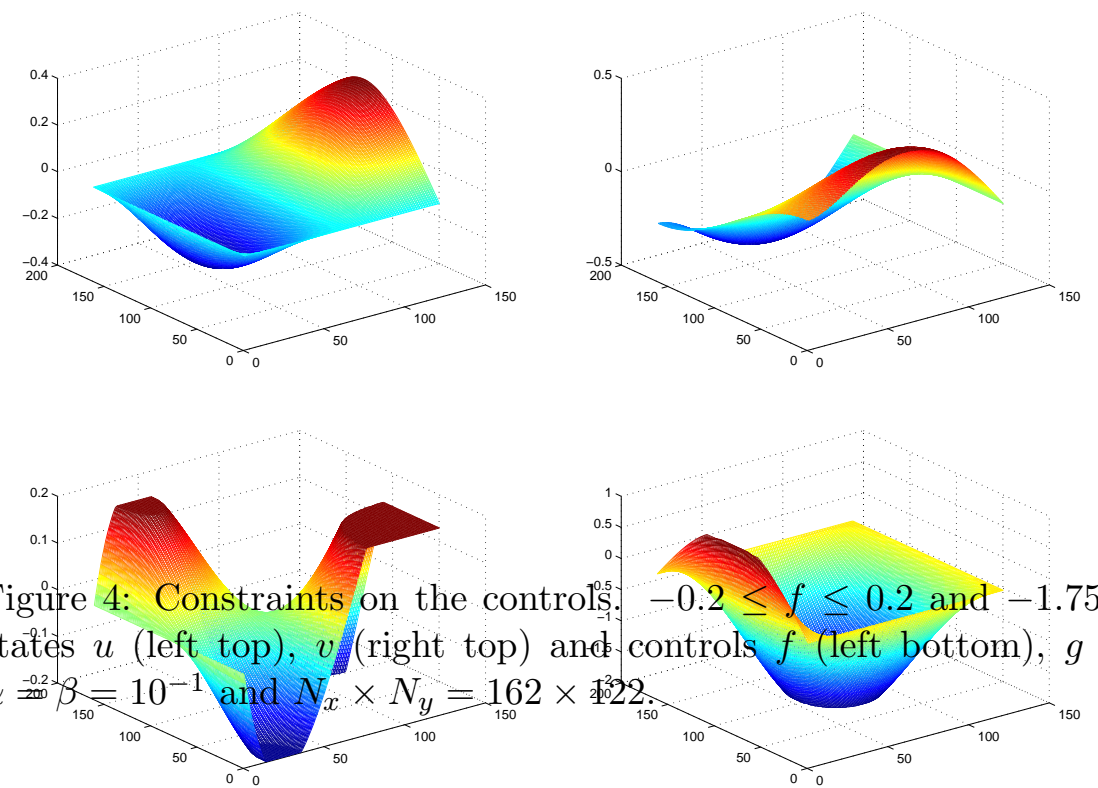

Figuire 4: Constraints on the controls. $-0.2 \leq f \leq 0.2$ and $-1.75 \leq g \leq 1$. The statés $u$ (left top), $v$ (right top) and controls $f$ (left bottom), $g$ (right bottom);



Since the $u$ and $v$ solution can be interpreted as the componets of a flow field, in Figure 6 , we depict the target vector functions $\mathbf{W}=(U, V)$ and the corresponding optimal solution $\mathbf{w}=(u, v)$.

The presence of constraints on the controls limit the tracking ability of the optimal control scheme. In Table 2, we report tracking errors for different choices of values of the control weights. We notice that only an almost negligible improvement in tracking is obtained chosing smaller values of the weights.

We investigate the convergence behaviour of the full multigrid scheme depending on the mesh size and on the optimization parameters. We focus on the case when constraints are active which requires additional computational effort. In Table 3, we report the observed values of the convergence factors for different meshes and different values of the optimization parameters. We obtain convergence factors that are almost mesh independent and are less sensitive to the choice of values of the $\alpha$ 

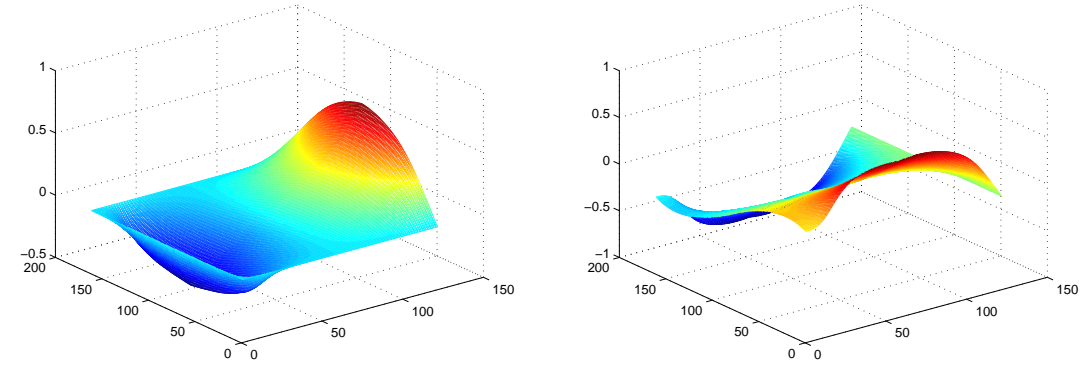

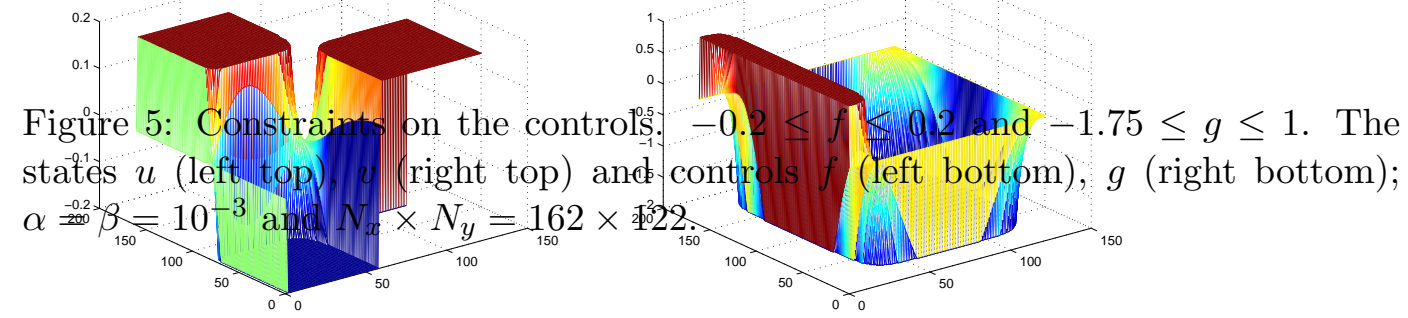
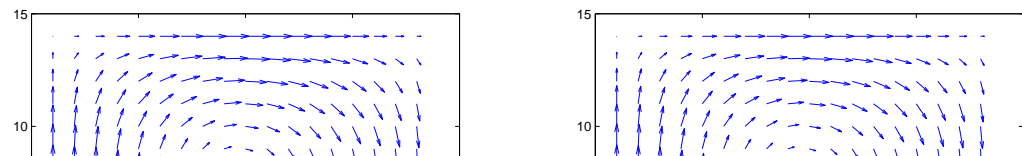

Figure 6: Tágét recirculatting flow $(U, W)$ (left) ánd optimall solution $(u, v)$ (right); $\alpha={ }_{5} \beta=10 \div 3$ and $N_{x} N_{y}=18 \times 14_{5}$

Table' 2: Discrete E ${ }^{2}$ norm of the tracking erròrs: Constraints on the controls.

\begin{tabular}{lll}
\hline$\alpha, \beta$ & $\|u-U\|_{L^{2}}$ & $\|u-V\|_{L^{2}}$ \\
\hline \hline & & \\
$10^{-2}, 10^{-2}$ & $4.03 \mathrm{E}-01$ & $2.70 \mathrm{E}-01$ \\
$10^{-2}, 10^{-4}$ & $4.02 \mathrm{E}-01$ & $2.66 \mathrm{E}-01$ \\
$10^{-4}, 10^{-2}$ & $4.03 \mathrm{E}-01$ & $2.71 \mathrm{E}-01$ \\
$10^{-4}, 10^{-4}$ & $4.02 \mathrm{E}-01$ & $2.67 \mathrm{E}-01$ \\
\hline
\end{tabular}


and $\beta$ weights.

Table 3: Convergence factors. Constraints on the controls.

\begin{tabular}{clcccc}
\hline$\alpha=\beta$ & $N_{x} \times N_{y}$ & $\rho_{u}$ & $\rho_{v}$ & $\rho_{p}$ & $\rho_{q}$ \\
& & & & & \\
\hline \hline & & & & & \\
$10^{-1}$ & $54 \times 41$ & $3.61 \mathrm{E}-01$ & $3.73 \mathrm{E}-01$ & $3.51 \mathrm{E}-01$ & $3.61 \mathrm{E}-01$ \\
& $162 \times 122$ & $3.50 \mathrm{E}-01$ & $3.64 \mathrm{E}-01$ & $3.27 \mathrm{E}-01$ & $3.50 \mathrm{E}-01$ \\
& & & & & \\
$10^{-3}$ & $54 \times 41$ & $7.14 \mathrm{E}-01$ & $8.08 \mathrm{E}-01$ & $7.40 \mathrm{E}-01$ & $7.14 \mathrm{E}-01$ \\
& $162 \times 122$ & $8.01 \mathrm{E}-01$ & $8.25 \mathrm{E}-01$ & $7.67 \mathrm{E}-01$ & $8.01 \mathrm{E}-01$ \\
& & & & & \\
$10^{-5}$ & $54 \times 41$ & $6.86 \mathrm{E}-01$ & $7.93 \mathrm{E}-01$ & $7.40 \mathrm{E}-01$ & $6.86 \mathrm{E}-01$ \\
& $162 \times 122$ & $8.08 \mathrm{E}-01$ & $8.51 \mathrm{E}-01$ & $7.86 \mathrm{E}-01$ & $8.08 \mathrm{E}-01$ \\
\hline
\end{tabular}

\section{Conclusion}

Control-constrained Cauchy-Riemann optimal control problems were presented and solved by a full multigrid scheme and staggered grids. We considered a smoothing procedure based on distributed-Gauss-Seidel iterations for the state and adjoint equations and a projected-gradient update for the controls. Results of numerical experiments were reported to validate the proposed formulation and to demonstrate the ability of the full multigrid scheme to solve efficiently control-constrained CauchyRiemann optimal control problems.

\section{References}

[1] M.M. Butt, A. Borzì, Formulation and multigrid solution of Cauchy-Riemann optimal control problems, submitted to CVS.

[2] A. Brandt, N. Dinar, Multigrid Solutions to Elliptic Flow Problems, Numerical Methods for PDEs, (1979) 53-147.

[3] A. Borzì and V. Schulz, Multigrid methods for PDE optimization, SIAM Review, 51 (2009), 361-395.

[4] A. Borzì, K. Morton, E. Suli, and M. Vanmaele,Multilevel solution of cell vertex Cauchy-Riemann equations, SIAM J. Sci. Comput., 18 (1997), 441-459.

[5] J.E. Dendy Jr and J.D. Moulton, Black Box Multigrid with coarsening by a factor of three, Numer. Linear Algebra Appl., 17 (2010), 577-598.

[6] L.C. Evans, Partial Differential Equations, Graduate Studies in Mathematics, American Mathematical Society, Providence, Rhode Island, vol. 19, 2002. 
[7] M. Ghil and R. Balgovind, A Fast Cauchy-Riemann Solver, Math. Comp., 33 (1979), 585-635.

[8] M.D. Gunzburger and H.-C. Lee, Analysis and approximation of optimal control problems for first-order elliptic systems in three dimensions, Applied Mathematics and Computation, 100 (1999), 49-70.

[9] G. Fix and M. Rose, A comparative study of finite element and finite difference methods for Cauchy-Riemann type equations, SIAM J. Numer. Anal., 22 (1985), 250-261.

[10] W. Hackbusch, Multi-grid Methods and Applications, Springer-Verlag, New York, 1985.

[11] K. Ito and K. Kunisch, Lagrange Multiplier Approach to Variational Problems and Applications, SIAM, 2008.

[12] J.L. Lions, Optimal Control of Systems Governed by Partial Differential Equations, Springer, Berlin, 1971.

[13] C.W. Oosterlee and F.J. Gaspar, Multigrid methods for the Stokes system, Computing in Science \& Engineering, 8 (2006), 34-43.

[14] S. Ta'asan, Canonical forms of multidimensional steady inviscid flows, Report No. 93-34, ICASE, Hampton, VA, 1993.

[15] F. Tröltzsch, Optimal control of partial differential equations: theory, methods and applications, AMS, 2010.

[16] U. Trottenberg, C. Oosterlee, and A. Schüller, Multigrid, Academic Press, London, 2001.

[17] D. Sidilkover, Factorizable schemes for the equations of fluid flow, Appl. Numer. Math., 41 (2002), 423-436.

[18] M. Vanmaele, K. W. Morton, E. Suli, and A. Borzì, Analysis of the cell vertex finite volume method for the Cauch-Riemann equations, SIAM J. Numer. Anal., 34 (1997), 2043-2062. 